\title{
Substantiation of the Absolute Velocity of the Soil Thrown by a Spherical Disc of the Roller of a Ridge Roller Planter
}

\author{
Evgeny Zykin ${ }^{1,}{ }^{*}$, Vladimir Kurdyumov ${ }^{1}$, and Svetlana Lazutkina ${ }^{1}$ \\ ${ }^{1}$ Department of Agrotechnologies, Machines and Life Safety, Ulyanovsk State Agrarian University, \\ Ulyanovsk, Russia
}

\begin{abstract}
For the finishing operation of the formation of the soil ridge of the required parameters and density simultaneously with sowing, a tillage roller has been designed, the novelty of which is confirmed by the patents of the Russian Federation No. 194330, No. 194348, No. 196712. The design of the roller assumes the regulation of the attackangles of the spherical discs and the amount of compression of the spring, because these parameters have a significant impact on the geometric dimensions of the formed ridge and the density of the soil in it. The proposed roller is mounted on the rear bead holder of a sowing gang of the ridgeroller planter. Moving along the surface of the field, the coulters of the ridge roller planter sow seeds on a wet compacted bed, the ridge formers form a loose soil ridge above the sown seeds, and the rollers compact the soil ridge. The speeds and forces acting on the spherical disc of the roller have been determined. It has been theoretically revealed that the absolute velocity of soil particles thrown by the edge of a spherical disc towards the longitudinal axis of symmetry of the soil ridge is maximum and depends on the angular speed of the spherical disc, its radius and angle of attack, as well as the frictional properties of the soil. The absolute speed of soil particles thrown from a specific point of the considered working body of the roller decreases when they approach the center of the spherical disc.
\end{abstract}

\footnotetext{
* Corresponding author: evg-zykin@yandex.ru
} 


\section{Introduction}

Having analyzedmany design options of rollers of row-crop planters, it was found that they are, to a greater extent, required for the destruction of soil clods and soil compaction before and after sowing on a flat surface of the field [1, 5-10].

Assessment indicators of ridge-forming rollers [1-8] show that there are currently unresolved issuesin the criteria for evaluating the geometric parameters and shapes of the surfaces of the working elements of rollers, namely, the method of determining the absolute speed of the soil thrown by spherical discs of the roller of the ridge roller planter. This parameter is the main one when justifying the distance between the spherical discs of rollers during the formation of soil ridges. Thus, additional theoretical substantiation of this parameter of the roller of the ridge roller planter is required.

\section{Materials and methods}

For the final operation - the formation of the soil ridge of the required parameters and density, simultaneously with sowing, we developed a roller, the novelty of which is confirmed by the patents of the Russian Federation No. 194330, No. 194348, No. 196712.

The design of the roller proposed by the authors (Fig. 1) incorporates the possibility of regulating the attack angle of the spherical discs and the compression force of the spring within certain limits since these parameters have a major impact on the geometric dimensions of the formed ridge and the density of the soil in it.

$$
\mathbf{A}-\mathbf{A}
$$
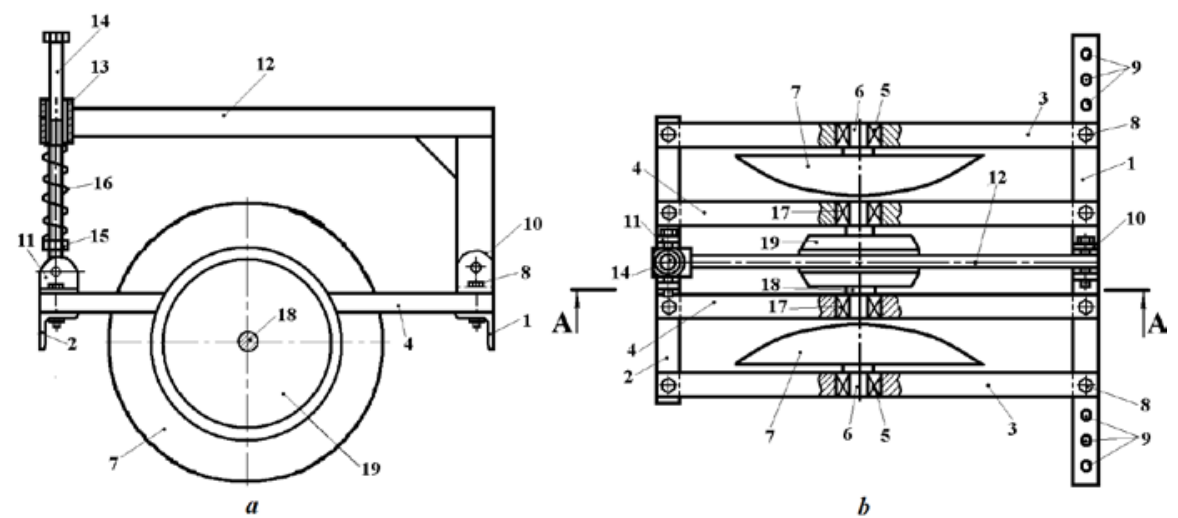

Fig. 1. Roller of the ridge roller planter: a - side view; $b$ - top view; 1, 2, 3 and 4 - front, rear, side and longitudinal beams, respectively; 5, 17 - bearings; 6 - half-shafts; 7 - spherical discs; 8 - bolts; 9 holes; 10, 11 and 12 - bearing, supporting and L-shaped brackets, respectively; 13 - guide sleeve; 14 rod; 15 - nut; 16 - spring; 18 - axis; 19 - press wheel 
Moving along the surface of the field, the openers of the ridge roller planter sow seeds, the ridge formers form a loose ridge of soil above the sown seeds, and the rollers compact this ridge of the soil (Fig. 2).

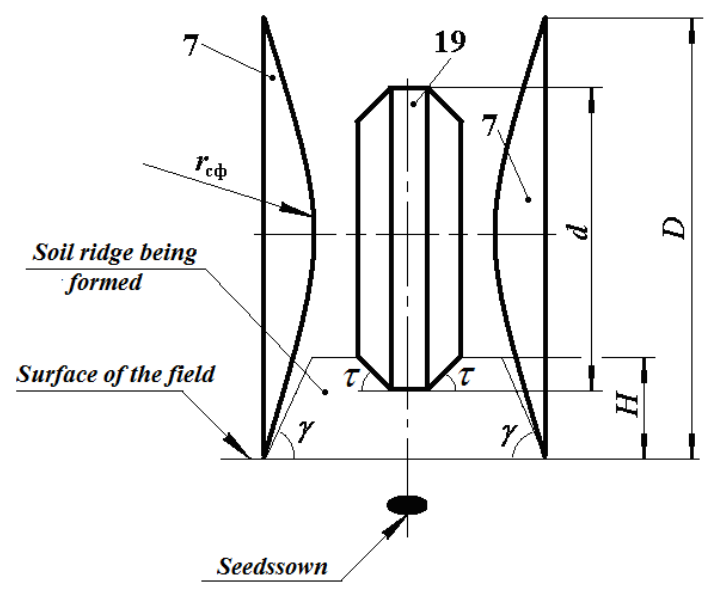

Fig. 2. The process of compaction of a loose ridge of soil with a roller: $r_{\mathrm{c} \phi}-$ sphere radius of the spherical disc, $\mathrm{m} ; d$-diameter of the press wheel, $\mathrm{m} ; D$-diameter of the spherical disc, $\mathrm{m} ; \gamma$ - angle of natural slope of the soil, deg.; $\tau$ - angle of the conical part of the press wheel, deg.; $H$ - height of a soil ridge, $\mathrm{m}$

Such a sequence of operations with correctly substantiated technological modes and design parameters of the working bodies of the roller should provide optimal conditions for germination and subsequent development of cultivated plants.

\section{Research results}

When the ridge roller planter moves at a speed of $v_{\mathrm{c}}, \mathrm{m} / \mathrm{s}$, the spherical discs of the roller move progressively and rotationally (Fig. 3), and each soil particle interacting with the spherical disc performs a complex movement characterized by relative, transfer and absolute speeds [1]. 


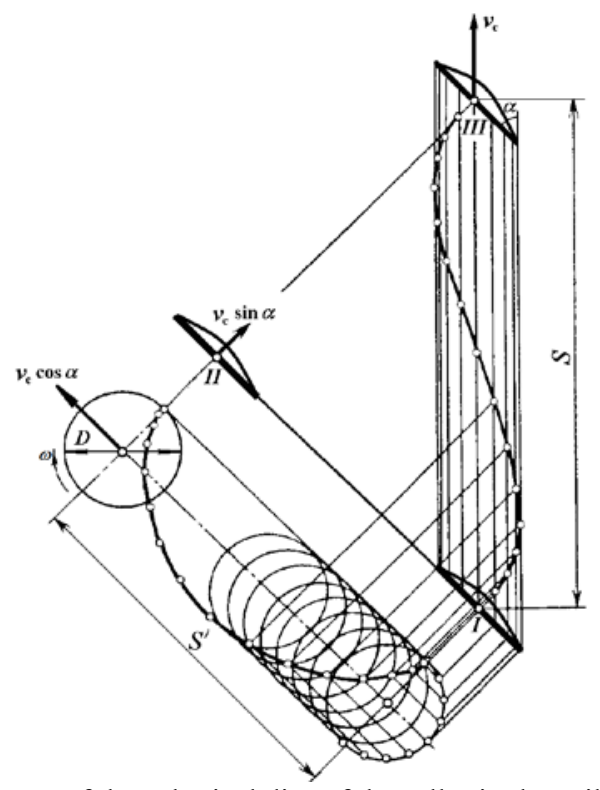

Fig. 3. Trajectory of movement of the spherical disc of the roller in the soil

The movement of the spherical disc in the soil, the working surface of which forms an angle $\alpha$ with the direction of movement of the ridge roller planter, from position $I$ to position $I I I$, will be broken down into two trajectories:

1) trajectory $I-I I$ - in the plane of a cutting edge of a spherical disc (rolling motion of a spherical disc without slip);

2) trajectory $I I-I I I$, perpendicular to trajectory $I-I I$ (progressive movement of a spherical disc without rotation).

The path $S^{\prime}, \mathrm{m}$, traveled by a spherical disc for one rotation from position $I$ to position II,

$$
S^{\prime}=\pi D
$$

Then the actual path $S, \mathrm{~m}$, in view of the angle of attack $\alpha$, deg., of a spherical disc:

$$
S=\frac{\pi D}{\cos \alpha} \text {. }
$$

Considering that the spherical disc rotates in the soil with slip [1], its angular speed of rotation, $\mathrm{c}^{-1}$,

$$
\omega=\frac{2 v_{\mathrm{c}} \cos \alpha}{D(1+\eta)}
$$

where $v_{\mathrm{c}}$ - speed of a ridge roller planter in the process of sowing, $\mathrm{m} / \mathrm{s} ; \eta-$ slip coefficient of a spherical disc in the soil. 
Slip coefficient:

$$
\eta=\frac{S-S^{\prime}}{S^{\prime}}=\frac{\frac{\pi D}{\cos \alpha}-\pi D}{\pi D}=\frac{1-\cos \alpha}{\cos \alpha} .
$$

Substituting expression (4) into (3), we find an expression for calculating the angular velocity $\omega$ of rotation of a spherical disc:

$$
\omega=\frac{v_{\mathrm{c}} \cos ^{2} \alpha}{R},
$$

where $R$ - radius of a spherical disc, $\mathrm{m}$.

The angular velocity $\omega$ of a spherical disc in the soil depends on its angle of attack $\alpha$ and radius $\mathrm{R}$, as well as the speed of the roller [1], which is equal to the speed $v_{c}$ of the ridge roller planter.

The relative velocity $v_{r}$ of soil (particle $\mathrm{M}$ ) displacement is directed along the tangent plane to the convex surface of the spherical disc of the roller in the direction opposite to the direction of movement of the roller, and is deflected from this direction by an angle $\alpha$ [1] (Fig. 4).

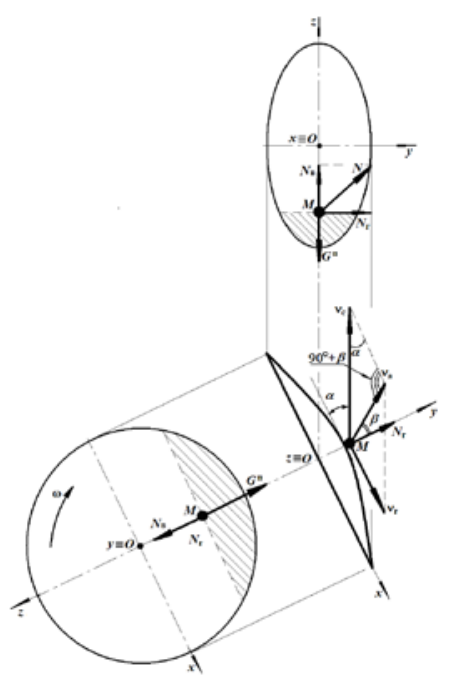

Fig. 4. Break down of velocities and forces on the plane of a spherical disc

The transport velocity $v_{\mathrm{c}}$ of a soil particle is directed along the roller movement trajectory [1].

The absolute velocity $v_{\mathrm{a}}$ of soil particles is deflected by a friction angle $\beta$ from the normal reaction $N$ of the soil. Normal reaction $N$, in its turn, is perpendicular to the plane tangent to the top of the convex surface of a spherical disc of the roller [1]. 
Having examined Fig.4, we can conclude that

$$
\frac{v_{a}}{\sin \alpha}=\frac{v_{\mathrm{c}}}{\sin \left(90^{\circ}+\beta\right)},
$$

where $\beta=\operatorname{arctg} f ; f$ - coefficient of friction between the soil and a spherical disc [1].

Taking into consideration that $\sin \left(90^{\circ}+\beta\right)=\cos \beta$, we write ratios between velocities:

$$
\frac{v_{a}}{\sin \alpha}=\frac{v_{\mathrm{c}}}{\cos \beta} \text {. }
$$

or

$$
v_{\mathrm{a}}=v_{\mathrm{c}} \frac{\sin \alpha}{\cos \beta} .
$$

Having derived the transport velocity $v_{c}$ from formula (5) and substituting it into (8), we determine the absolute velocity of the soil thrown by the edge of the spherical disc:

$$
v_{\mathrm{a}}=\frac{\omega r \sin \alpha}{\cos ^{2} \alpha \cos \beta} \text {. }
$$

where $r$ - variable distance from the center of the axis of rotation of the spherical disc to the soil particle in contact with the surface of the spherical disc, $\mathrm{m}$.

The maximum absolute speed of the moved soil will be at the cutting edges of the spherical disc, and the minimum value will be at the center of the spherical disc.

\section{Conclusion}

Analyzing expression (9), it can be stated that at $r=R$ the absolute speed of the soil, moved by the edges of each spherical disc of the roller towards the longitudinal axis of symmetry of the soil ridge, is maximum and depends on the angular velocity of the spherical disc, its radius and angle of attack, as well as the frictional properties. The maximum absolute speed of the thrown soil particles will be at the cutting edges of the spherical disc, and the minimum value of the absolute speed will be at the center of the spherical disc.

The project is carried out within the framework of the grant of the President of the Russian Federation for state support of young Russian scientists - Doctors of Science MD-2259.2020.8.

\section{References}

1. Zykin, E.S. Development and substantiation of technology and mechanization means of ridge cultivation of row crops: spec. 05.20.01 - Technologies and means of agricul- 
tural mechanization: dissertation ... Doctor of Technical Sciences / Zykin Evgeny Sergeevich (Ufa). - Ulyanovsk, 2017.- 637 p.

2. Zykin, E. Process modeling of the first interrow cultivation in laboratory conditions / Evgeny Zykin, Vladimir Kurdyumov, Svetlana Lazutkina, Oleg Dmitriev // E3S Web of Conferences 193, 01041 (2020). ICMTMTE 2020.

3. Zykin, E. The experimental determination of the diameter of a flat disc in a ridge seeder / Evgeny Zykin, Vladimir Kurdyumov, Svetlana Lazutkina, Sergey Albutov // IOP Conf. Series: Ma-terials Science and Engineering 971 (2020) 052055. ICMTMTE 2020 .

4. Zykin, E. Modeling of the sowing process of row crops in laboratory conditions / Evgeny Zykin, Vladimir Kurdyumov, Sergey Albutov, Oleg Dmitriev // E3S Web of Conferences 193, 01040 (2020). ICMTMTE 2020.

5. Erzamaev, M.P. Improving the efficiency of using arable machines / M.P. Erzamaev, D.S. Sazonov, E.O. Salomatov // Innovative achievements of science and technology of the agro-industrial complex: collection of scientific papers of the International scientific and practical conference. - Kinel: Samara State Agricultural Academy, 2017.pp. 689-692.

6. Akramkhanov, A. Technology of planting crops along the ridges / A. Akramkhanov // TECHNOLOGIES \& BEST PRACTICES FACTSHEET. - URL: http://www.cacilm.org/articles/detail/493 (access date 05/09/2021).

7. Babitskiy, L. Results of research of working bodies with increased reliability of tillage and sowing machines / Leonid Babitskiy, Vadim Moskalevich, and Aleksandr Belov // E3S Web of Conferences 193, 01042 (2020). ICMTMTE 2020.

8. Belousov, S.V. On the problem of interaction of the tillage working body with the soil / Sergey V. Belousov and Evgeny E. Samurganov // E3S Web of Conferences 193, 01062 (2020). ICMTMTE 2020.

9. GOST R 54783-2011. Agricultural machinery tests. Introduced on 2011-12-13. Moscow: Publishing house of standards, 2011. - 23 p. (Approved and brought into effect by the Order of the Federal Agency for Technical Regulation and Metrology dated December 13, 2011 No. 995-st, Moscow).

10. GOST R 54784-2011. Agricultural machinery tests. Methods for assessing technical parameters. Introduced on 2012-03-01. - Moscow: Standards Publishing House, 2012. - 23 p. (Approved and brought into effect by the Order of the Federal Agency for Technical Regulation and Metrology dated December 13, 2011 No. 996-st, Moscow). 